\title{
Independence of Movement Preparation and Movement Initiation
}

\author{
Adrian M. Haith, ${ }^{1}$ Jina Pakpoor, ${ }^{1,3}$ and John W. Krakauer ${ }^{1,2}$ \\ Departments of ${ }^{1}$ Neurology and ${ }^{2}$ Neuroscience, Johns Hopkins University, Baltimore, Maryland 21287, and ${ }^{3}$ School of Clinical Medicine, University of \\ Cambridge, Cambridge, CB2 0SP, United Kingdom
}

Initiating a movement in response to a visual stimulus takes significantly longer than might be expected on the basis of neural transmission delays, but it is unclear why. In a visually guided reaching task, we forced human participants to move at lower-than-normal reaction times to test whether normal reaction times are strictly necessary for accurate movement. We found that participants were, in fact, capable of moving accurately $\sim 80 \mathrm{~ms}$ earlier than their reaction times would suggest. Reaction times thus include a seemingly unnecessary delay that accounts for approximately one-third of their duration. Close examination of participants' behavior in conventional reaction-time conditions revealed that they generated occasional, spontaneous errors in trials in which their reaction time was unusually short. The pattern of these errors could be well accounted for by a simple model in which the timing of movement initiation is independent of the timing of movement preparation. This independence provides an explanation for why reaction times are usually so sluggish: delaying the mean time of movement initiation relative to preparation reduces the risk that a movement will be initiated before it has been appropriately prepared. Our results suggest that preparation and initiation of movement are mechanistically independent and may have a distinct neural basis. The results also demonstrate that, even in strongly stimulus-driven tasks, presentation of a stimulus does not directly trigger a movement. Rather, the stimulus appears to trigger an internal decision whether to make a movement, reflecting a volitional rather than reactive mode of control.

Key words: movement initiation; movement preparation; reaching; reaction time; volitional movement

\section{Significance Statement}

The reaction time, i.e., how quickly we can initiate a movement in response to a stimulus, is important for daily activities such as driving and is also a critical tool in neuroscience, used to probe a multitude of cognitive functions. However, there remains a surprising lack of basic understanding about exactly what determines reaction times, even for simple movements such as reaching to a target. We show that the reaction time for a reaching movement does not reflect the moment that the movement becomes ready to execute. Instead, the reaction time is determined by a separate initiation process. These findings suggest a distinct neural basis for preparation and initiation of movement and provide an explanation for the sluggishness of typical reaction times.

\section{Introduction}

Each time we make a movement, a series of processes must occur in our brain that include perceiving the cue, deciding on highlevel movement goals (e.g., where to move), and determining the low-level details of the movement that will achieve those goals (e.g., which muscles to contract and when; Haith et al., 2015a; Smeets et al., 2015; Wong et al., 2015). It is commonly assumed

Received Aug. 28, 2015; revised Jan. 14, 2016; accepted Jan. 23, 2016

Author contributions: A.M.H. and J.W.K. designed research; A.M.H. and J.P. performed research; A.M.H. analyzed data; A.M.H. and J.W.K. wrote the paper.

This work was supported by National Science Foundation Grant 1358756. We thank Aaron Wong and Nicole Kett for experimental assistance.

Correspondence should be addressed to Adrian M. Haith, Johns Hopkins University School of Medicine, 210 Carnegie, 550 North Wolfe Street, Baltimore, MD 21287. E-mail: adrian.haith@jhu.edu.

DOI:10.1523/JNEUROSCI.3245-15.2016

Copyright $\odot 2016$ the authors $\quad 0270-6474 / 16 / 363007-10 \$ 15.00 / 0$ that a movement will be initiated as soon as these movement preparation steps are complete (Donders, 1969; Rosenbaum, 1980; Erlhagen and Schöner, 2002; Cisek, 2006; Smeets et al., 2015). Consequently, the reaction time (RT) has long been thought to directly reflect how long it takes to prepare a movement. Here, we challenge this view.

Reaching to a visually presented target typically incurs an RT of $\sim 200-300 \mathrm{~ms}$ (Welford, 1980). This is far longer than might be expected on the basis of neural transmission delays (Carpenter, 1999). However, it is well known that goal-directed responses can occur at much shorter latencies if participants are required to correct an ongoing movement. Humans react to displacements of a target (van Sonderen and Denier van der Gon, 1991; Gritsenko et al., 2009) or cursor (Day and Lyon, 2000; Franklin and Wolpert, 2008) during a movement with latencies of $\sim 150 \mathrm{~ms}$. Mechanical perturbations of the arm elicit goal-directed responses in 
as little as $100 \mathrm{~ms}$ (Pruszynski et al., 2008; Yang et al., 2011; Nashed et al., 2014).

The reason for the discrepancy between the latency of online feedback corrections and the latency of movement initiation remains unknown. One possible explanation is that participants always generate a response as soon as possible but that initiating a movement takes longer because it entails additional processing steps (Smeets et al., 2015). Another possibility is that participants are capable of initiating an accurate movement much earlier but, for some reason, delay their initiation. For example, it is well known that simple RTs (i.e., initiating a pre-prepared movement in response to a "go" cue) can be shortened by $\sim 70 \mathrm{~ms}$ if participants are startled by a loud acoustic stimulus at the time of the go cue (Valls-Solé et al., 1999; Carlsen et al., 2004).

We performed two experiments to more clearly characterize the relationship between preparation and initiation of movements. Although movement preparation cannot be measured directly, it is possible to establish its progression indirectly by forcing participants to move at lower-than-normal RTs and assessing the accuracy of their movements as a function of the imposed RT (Schouten and Bekker, 1967; Ghez et al., 1997; Haith et al., 2015a). Using this approach, we found that reaching movements to a visual target become accurately prepared in $\sim 130 \mathrm{~ms}, \sim 80 \mathrm{~ms}$ earlier than typical RTs. We further sought to more precisely determine the relationship between the timing of movement preparation and movement initiation in a given trial. One possibility is that movement initiation always occurs at a fixed delay after movement preparation (Luce, 1986). Alternatively, there may be no causal relationship between preparation and initiation; the timings of these two events might be determined independently. In this case, delaying the mean time of movement initiation could serve to avoid the risk of initiating a movement before it has been prepared.

\section{Materials and Methods}

A total of 22 human participants were recruited for this study (10 in Experiment 1 and 12 in Experiment 2). All participants had no known neurological disorder and provided written consent before participation. All procedures were approved by the Johns Hopkins University School of Medicine Institutional Review Board.

Experimental setup. Participants sat at a glass-surfaced table with their arm supported by a plastic cradle mounted on pressurized air jets, allowing frictionless planar movements of their arm. Participants viewed a display through a mirror positioned horizontally above their arm (Fig. $1 A$ ). The position of the hand was tracked at $130 \mathrm{~Hz}$ by a magnetic tracking device (Flock of Birds; Ascension) and was displayed veridically via the mirror as a small, blue cursor ( $5 \mathrm{~mm}$ diameter). Participants were required to move their hands rapidly to guide a cursor through a presented target (25 mm diameter) from a central start location $(10 \mathrm{~mm}$ diameter). There were eight possible target locations, distributed equally around the start location at a distance of $80 \mathrm{~mm}$ and indicated by a small (10 mm diameter) gray circle. On-screen feedback graphically indicated the peak tangential velocity attained on each trial, and participants were instructed to ensure that this exceeded a threshold corresponding to 0.9 $\mathrm{ms}^{-1}$ on each trial.

Experimental tasks. Ten participants (aged $23.6 \pm 6.4$ years; five women) were recruited for Experiment 1 . These participants experienced two conditions. In the first condition, the Free RT condition (Fig. $1 B$, left), participants were instructed to initiate a movement as quickly as possible after the target was presented. To minimize ambiguity about the time of target presentation (which tends to increase RTs; Frith and Done, 1986), a predictable sequence of auditory tones (four tones spaced $500 \mathrm{~ms}$ apart) cued the time of target presentation. Participants performed two blocks of 96 trials.
In the second condition, the Forced RT condition (Fig. 1B, right), participants were trained to initiate their movement synchronously with the onset of the fourth tone. Movement initiation time was determined online as the time at which the tangential velocity first exceeded $0.02 \mathrm{~ms}^{-1}$. If participants failed to initiate their movement within $75 \mathrm{~ms}$ of this time, on-screen text indicated "Too Early" or "Too Late" as appropriate. The first block (50 trials) allowed participants to practice the timing of their initiation. In this block, the target was visible from the onset of the first tone, allowing $1500 \mathrm{~ms}$ for participants to prepare an appropriate movement. In subsequent blocks $(3 \times 106)$, the amount of preparation time $\left(T_{\mathrm{P}}\right)$ was varied from trial to trial by presenting the target at varying times (uniformly distributed between 0 and $300 \mathrm{~ms}$ ) prior to the fourth tone (96 of 106 trials). The delay between the stimulus presentation and the time of movement initiation in each trial defined an effective RT for that trial. A subset of eight trials were "catch" trials in which no target ever appeared but participants were still required to move. These catch trials discouraged participants from simply waiting until the target appeared before initiating a movement, in which case behavior would be very similar to that in the Free RT condition. The first two trials of each of these blocks served as warmup trials in which the target appeared at the onset of the first tone.

Twelve new participants (aged $23.5 \pm 4.9$ years; seven women) were recruited for Experiment 2. Participants experienced two conditions in this experiment. One condition, the Forced RT condition, was identical to that in Experiment 1, except that participants performed seven blocks of 106 trials. An additional condition, the Pressured RT condition, was similar to the Free RT condition in Experiment 1, except that a deadline was imposed on the time of movement initiation. The deadline was visually cued to participants by presenting a shrinking green disc within the presented target (the target itself remained visible as a black circle). If participants failed to initiate their movement before the green disc shrank to zero, the target disappeared, the cursor turned red, and an unpleasant rasping sound was played. The rate of the target shrinking was varied across blocks to yield a progressively more stringent deadline: no deadline in Block 1 (making it equivalent to the Free RT condition of Experiment 1), deadlines of 900, 400, 300, 233, 208, and 186 in Blocks 2-7, and no deadline in Block 8 . The order in which participants experienced the two conditions (Forced RT and Pressured RT) was counterbalanced across the 12 participants. One participant in Experiment 2 exhibited erratic behavior from Block 2 onward in the Pressured RT condition (having a mean RT $>2.5$ SDs below the mean across subjects) and was therefore excluded from subsequent analysis to avoid distorting the results. Nevertheless, including this participant in our analyses did not qualitatively alter our conclusions.

Data analysis. Raw hand position data were smoothed and differentiated using a second-order Savitzky-Golay filter with a half-width of $54 \mathrm{~ms}$. Movement onset was detected based on the first time that the tangential velocity of the hand exceeded $0.02 \mathrm{~ms}^{-1}$. From this time, we subtracted the mean delays in our system (measured to be $100 \mathrm{~ms}$ ) to obtain an estimate of the true time of movement initiation. In all conditions, the RT (or effective RT, in the Forced RT condition) was determined as the delay between the time of stimulus presentation and the time of movement initiation. Free RT and Pressured RT trials in which the RT was less than $-200 \mathrm{~ms}$ (i.e., >200 ms before presentation of the target) or $>500 \mathrm{~ms}$ were excluded from additional analysis. Overall, $0.4 \%$ of all trials were excluded on these grounds.

Movement direction in each trial was assessed based on the direction of the velocity vector of the hand $100 \mathrm{~ms}$ after movement onset. A movement was deemed to have been initiated accurately if the initial direction of movement was within $22.5^{\circ}$ of the target direction. Otherwise, the trial was considered to be an error. The probability of initiating an accurate movement at a given RT in the Forced RT condition was estimated based on the proportion of accurately initiated movements within a $20 \mathrm{~ms}$ window around that RT, yielding a speed-accuracy tradeoff (Wickelgren, 1977), also closely related to the concept of a tachometric curve (Stanford et al., 2010). To more compactly quantify this speed-accuracy tradeoff, we assumed a sim- 
A
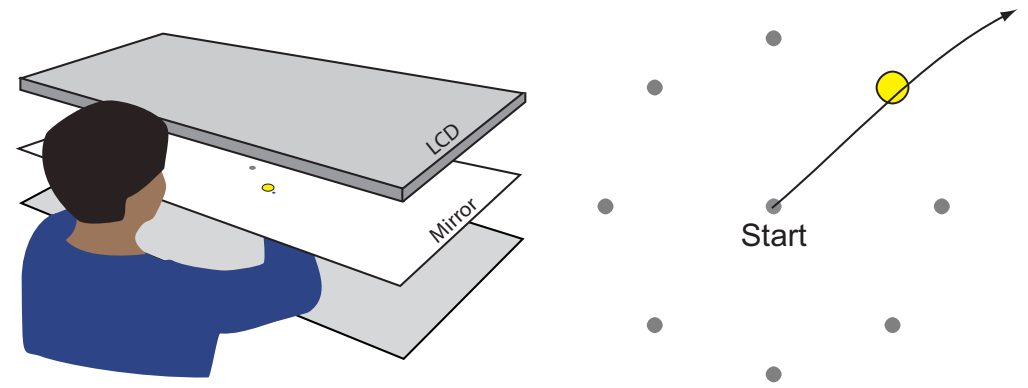

B
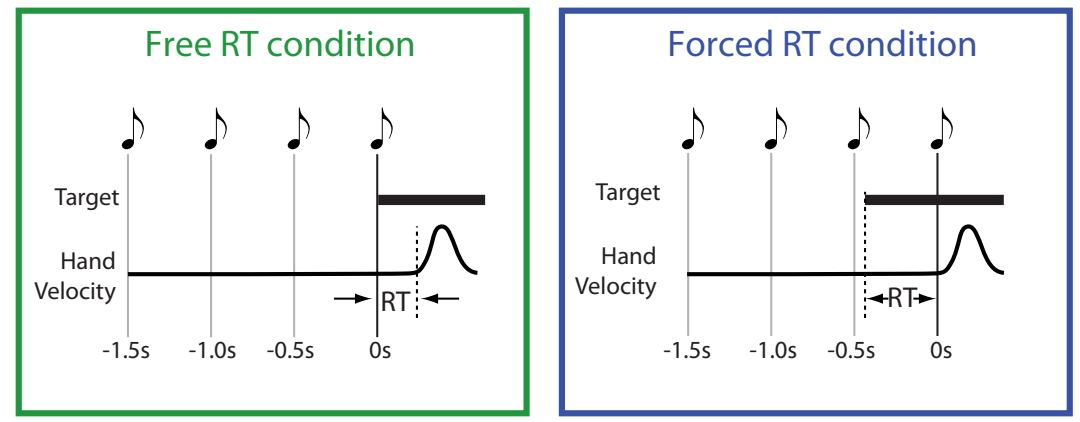

C

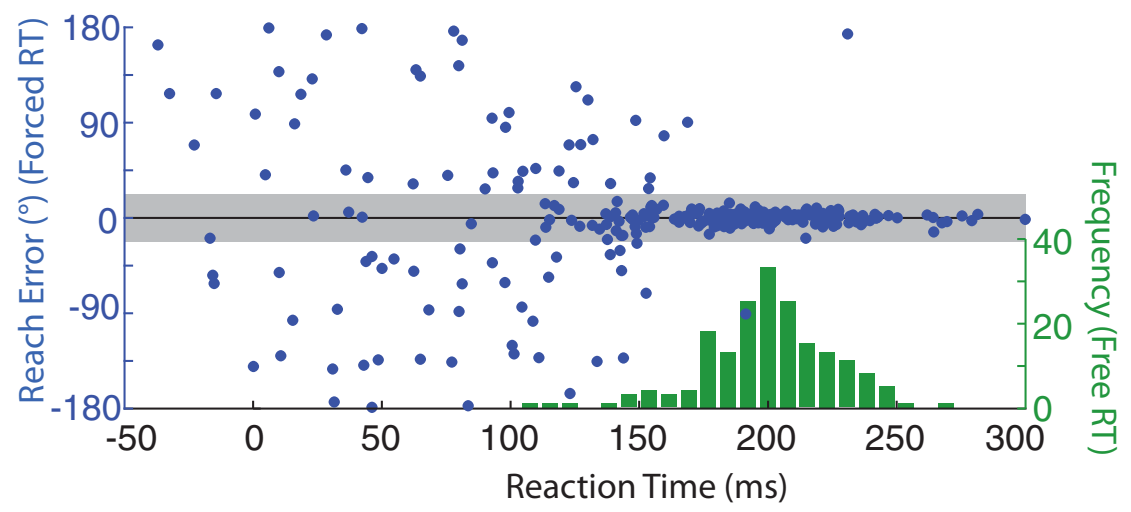

Figure 1. Experimental design and example data. $\boldsymbol{A}$, Experimental setup. Participants made planar reaching movements to targets presented via a mirrored display. $\boldsymbol{B}$, Free RT condition (left) and Forced RT condition (right). In the Free RT condition (left), a single target appeared at an unknown location at a predictable time (cued by a series of 4 tones), and participants were instructed to move as soon as the target appeared. In the Forced RT condition (right), participants were instructed to move synchronously with the fourth tone. RT was manipulated by varying the time that the target was presented relative to the time of movement onset. $\boldsymbol{C}$, Behavior of a representative participant. Green histogram indicates the distribution of RTs in the Free RT condition (both correct and incorrect movements). Blue circles indicate RT ( $x$-axis) and directional error ( $y$-axis) for each trial in the Forced RT condition. Gray region indicates the range of directions considered to be accurate.

ple model in which a single preparation event occurred at a stochastic time $T_{\mathrm{P}} \sim N\left(\mu_{P}, \sigma_{P}^{2}\right)$. We assumed that movements initiated before $T_{\mathrm{P}}$ were directed randomly, whereas movements initiated after $T_{\mathrm{P}}$ were initiated accurately toward the target. We also allowed for the fact that participants were not $100 \%$ accurate even at long RTs through a parameter $\alpha$ that defined the participant's asymptotic accuracy, which we fixed at $\alpha=0.95$. The probability of the movement in trial $i$ being accurate $\left(H^{i}=1\right)$ given that it was initiated at time $\mathrm{RT}^{i}$ was then given by:

$$
\begin{aligned}
p\left(H^{i} \mid \mathrm{RT}^{i} ; \mu_{P}, \sigma_{P}^{2}\right) & =\alpha p\left(\mathrm{RT}^{i} \geq \mathrm{T}_{\mathrm{P}}^{i}\right)+\frac{1}{8} p\left(\mathrm{RT}^{i}<\mathrm{T}_{\mathrm{P}}^{i}\right) \\
& =\alpha \Phi\left(\mathrm{RT}^{i} \mid \mu_{P}, \sigma_{P}^{2}\right)+\frac{1}{8}\left(1-\Phi\left(\mathrm{RT}^{i} \mid \mu_{P}, \sigma_{P}^{2}\right)\right)
\end{aligned}
$$

where $\Phi$ is the cumulative normal distribution. The log likelihood for each individual trial was therefore given by:

$$
\begin{gathered}
\mathrm{LL}^{i}\left(H^{i} \mid \mathrm{RT}^{i}, \mu_{P}, \sigma_{P}^{2}\right)=H^{i} \log \left[\frac{1}{8}\right. \\
\left.+\left(\alpha-\frac{1}{8}\right) \Phi\left(\mathrm{RT}^{i} \mid \mu_{P}, \sigma_{P}^{2}\right)\right] \\
+\left(1-H^{i}\right) \log \left[\frac{7}{8}\right. \\
\left.-\left(\alpha-\frac{1}{8}\right) \Phi\left(\mathrm{RT}^{i} \mid \mu_{P}, \sigma_{P}^{2}\right)\right] .
\end{gathered}
$$

The likelihoods for each trial were summed to obtain the overall log likelihood. We identified the distribution of the timing of movement preparation via the parameters $\mu_{P}$ and $\sigma_{P}$ that maximized this overall likelihood. Given these estimates, obtained independently for each participant based on their behavior in the Forced RT condition, we predicted the expected error rate in the Free RT condition (Experiment 1) or Pressured RT condition (Experiment 2) using Equation 1 and based on the exact RTs that participants generated in those blocks.

We also estimated the time of preparation based on how the distribution of initial reach directions evolved as RT increased. For simplicity, we considered reach direction to follow a discrete distribution over the eight potential target directions. For movements initiated before they were prepared $\left(\mathrm{RT}<T_{\mathrm{P}}\right.$ ), we assumed that reach direction $d=1 \ldots 8$ followed a specific guess distribution, $\mathbf{q}_{\text {guess }}(d)$, which we estimated from catch trials (in which no target ever appeared). For movements initiated after they became appropriately prepared $\left(\mathrm{RT}>T_{\mathrm{P}}\right)$, we assumed that initial reach direction followed a uniform distribution, $\mathbf{q}_{\text {targ }}(d)$, reflecting the distribution of actual target locations. The overall distribution of movements at a given RT was then given by a mixture of these two distributions:

$$
\begin{aligned}
& \mathbf{q}(d \mid \mathrm{RT})=(1-\Theta(\mathrm{RT})) \mathbf{q}_{\text {guess }}(d) \\
&+\Theta(\mathrm{RT}) \mathbf{q}_{\mathrm{targ}}(d) .
\end{aligned}
$$

We pooled data from all 10 participants in Experiment 1 and used a maximum likelihood approach to estimate $\Theta(t)$ in two ways: (1) nonparametrically, using data in a sliding window of width $50 \mathrm{~ms}$ to estimate $\Theta(t)$ for each possible value of $t$ between 0 and $300 \mathrm{~ms}$; and (2) parametrically, assuming that $\Theta(t)=\Phi\left(t \mid \mu_{P}, \sigma_{P}^{2}\right)$, as above.

To visualize the distribution of RTs in the Free RT and Pressured RT conditions, we used kernel density estimation to estimate the distribution, using Gaussian kernels with an SD of $10 \mathrm{~ms}$. To visualize the distribution of reach directions in the Forced RT condition, we used a similar kernel density estimation procedure using von Mises kernels with a kernel width of $5^{\circ}$.

\section{Results}

Movement initiation is delayed by $80 \mathrm{~ms}$ relative to movement preparation

In Experiment 1, participants made planar reaching movements from a central start position toward one of eight potential targets (Fig. 1A). Participants first performed an RT task in which they were instructed to move as soon and as accurately as possible after 
A

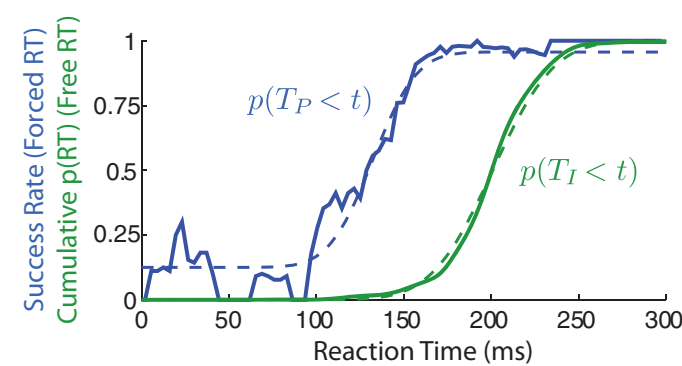

C

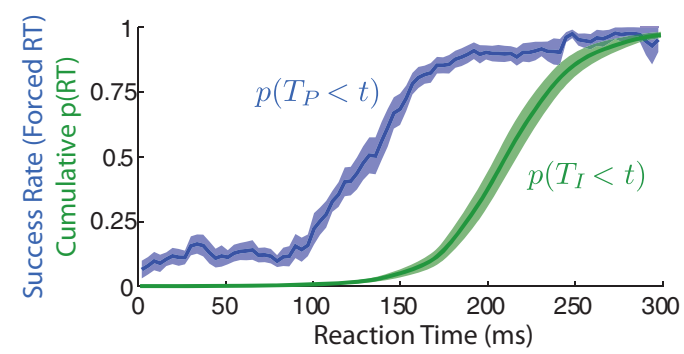

E

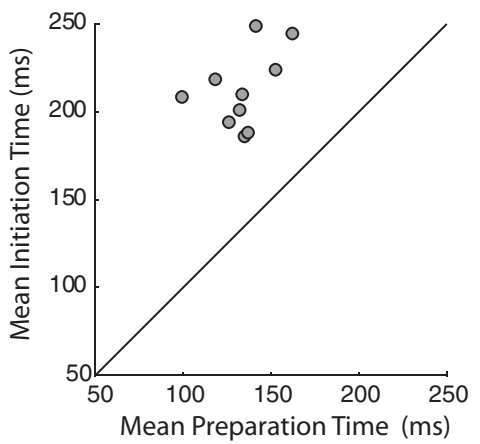

B

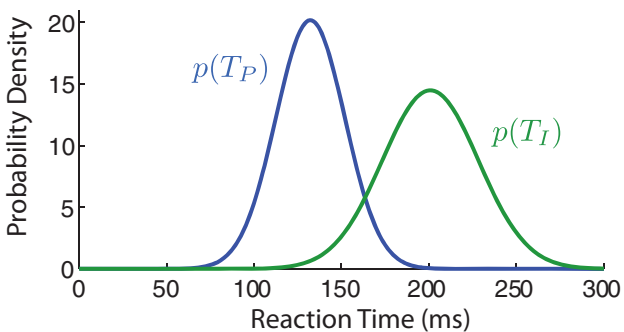

D

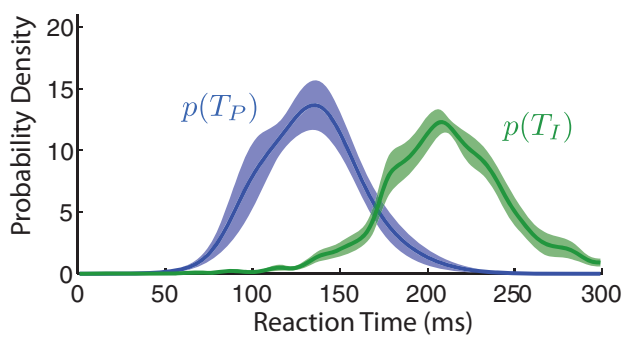

$\mathbf{F}$

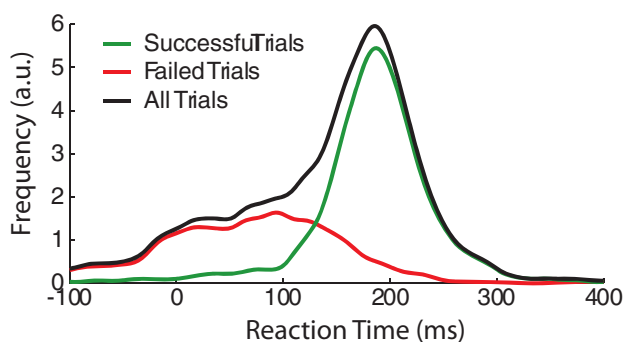

Figure 2. Movement initiation lags movement preparation. $\boldsymbol{A}$, Blue line, Moving average of the probability that a movement is successful for a given RT in the Forced RT condition, which corresponds to the cumulative probability. Green line, Cumulative distribution of RTs in the Free RT condition. Dashed lines indicate model fits based on maximum likelihood estimation of $T_{\mathrm{p}}$ and $T_{\text {I }}$ distributions. $\boldsymbol{B}$, Estimated distributions of $T_{\mathrm{p}}$ and $T_{1}$ for the same example participant as shown in Figure 1 . C, $\boldsymbol{D}$, Same as $\boldsymbol{A}$ and $\boldsymbol{B}$ but showing mean behavior across 10 participants. Shaded regions indicate \pm SEM. $\boldsymbol{E}$, Mean $T_{1}$ versus estimated mean $T_{\mathrm{p}}$. Each point represents a single participant. $\boldsymbol{F}$, Overall distribution of effective RTs in the Forced RT condition (across all participants). Green, Successful trials; red, failed trials; black, all trials.

they saw the target (Free RT condition; Fig. 1B, left). The distribution of RTs exhibited by a representative participant is shown in Figure $1 C$ (green histogram). This participant had a mean \pm SD RT of $201 \pm 28 \mathrm{~ms}$. This participant's mean RT was slightly shorter than the average mean RT across all participants, which was $212 \pm 22 \mathrm{~ms}$.

In a second phase of the experiment, we sought to assess the time course of movement preparation. We asked whether the RTs participants took in the Free RT condition were necessary to move accurately. We manipulated the amount of $T_{\mathrm{P}}$ available before each movement by training the participant to initiate their movement at a specific time during each trial and varying the time at which the target was presented relative to movement onset (Forced RT condition; Fig. 1B, right; for details, see Materials and Methods). This condition revealed that the same participant who previously had a mean RT of $201 \mathrm{~ms}$ was in fact capable of consistently reaching toward the correct target even when their effective RT (i.e., the time between target presentation and movement onset) was as low as $170 \mathrm{~ms}$ (Fig. 1C). Only the very fastest RTs in the Free RT condition approached this value (10th RT percentile $=177 \mathrm{~ms}$ ). The majority of trials in the Free RT condition had RTs that were much greater, in some cases by up to 60 $\mathrm{ms}$ (90th RT percentile in the Free RT condition $=230 \mathrm{~ms}$ ). All 10 participants showed the same pattern of being consistently accurate in the Forced RT condition at effective RTs well below the RTs they exhibited in the Free RT condition.

To better understand the relationship between behavior in the Free RT and Forced RT conditions, we considered a simple model of movement preparation and initiation. We assumed that, in any trial, two distinct events occur. First, a movement becomes appropriately prepared at time $T_{\mathrm{P}}$ after presentation of the stimulus. Second, the movement is initiated at time $T_{\mathrm{I}}$. Although we could not directly observe $T_{\mathrm{P}}$, we could infer its distribution from behavior in the Forced RT condition. Assuming that participants move accurately if $T_{\mathrm{I}} \geq T_{\mathrm{P}}$, and randomly if $T_{\mathrm{I}}<T_{\mathrm{P}}$, the probability that a movement initiated at a particular time $t$ is accurate is given by the cumulative distribution of $T_{\mathrm{P}}, p\left(T_{\mathrm{P}}<t\right)$. This distribution can be estimated from data in the Forced RT condition, whereas the distribution of $T_{\mathrm{I}}$ can be obtained directly from the Free RT condition. Figure $2 A$ shows the estimated cumulative distributions of $T_{\mathrm{P}}$ and $T_{\mathrm{I}}$ for the same representative participant as before. Comparing these two curves reveals a significant disparity between the timing of these two events. To enable a quantitative comparison between $T_{\mathrm{P}}$ and $T_{\mathrm{I}}$, we used maximum likelihood estimation to parametrically estimate the distribution of $T_{\mathrm{P}}$ for each participant, assuming a Gaussian distribution (Fig. $2 B$; for details, see Materials and Methods). For the representative participant, this analysis yielded an estimated mean $T_{\mathrm{P}}$ of $132 \mathrm{~ms}$, 

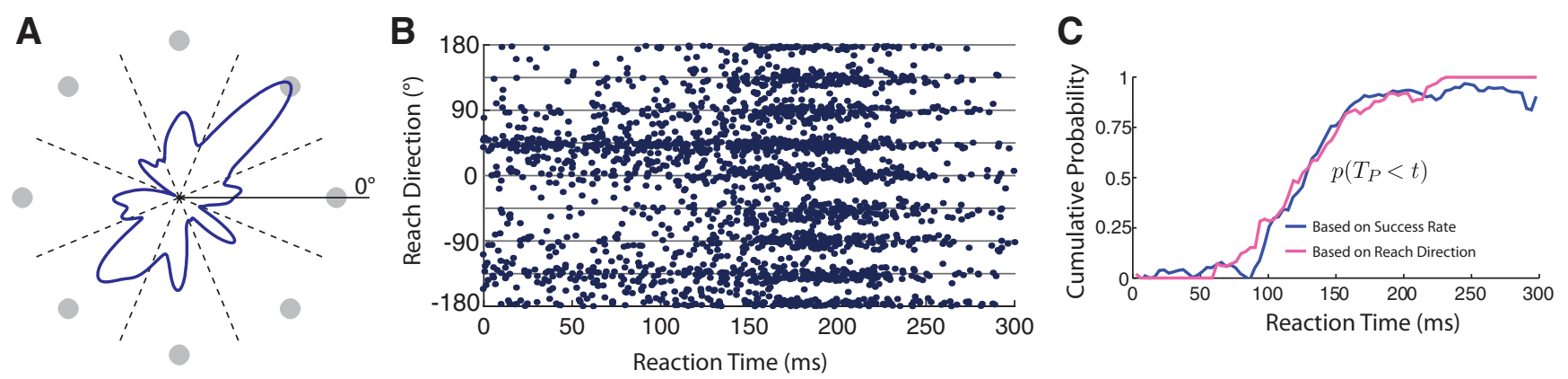

Figure 3. Distribution of guesses and alternative estimate of $T_{\mathrm{p}}$. $\boldsymbol{A}$, Circular histogram showing the distribution of initial movement directions in catch trials in the Forced RT condition in Experiment 1. Gray circles indicate potential target locations. Dashed black lines indicate classification boundaries used to obtain the discrete distribution $\mathbf{p}_{\text {guess }}(d), \boldsymbol{d}=1$... 8 . $\boldsymbol{B}$, Reach direction as a function of effective RT in the Forced RT condition in Experiment 1. Each point represents a single trial. Gray lines indicate target directions. $\boldsymbol{C}$, Estimated time course of movement preparation, $p\left(T_{\mathrm{P}}\right.$ $<t$ ). Blue line, Estimate based on improving success rate with RT, as in Figure 2, $A$ and $C$. Pink line, Estimate based on the changing distribution of initial reach angles, seen in $B$. All plots in this figure are generated from data pooled across participants.

with an SD of $19 \mathrm{~ms}$. Comparing this with the observed mean of $T_{\mathrm{I}}$, we estimated that this participant exhibited, on average, a delay of $68 \mathrm{~ms}$ between the time at which their movements became appropriately prepared and the time at which they were initiated. Similar behavior was identified for all 10 participants (Fig. $1 C-E$ ), with a mean time of preparation consistently lower than the mean time of movement initiation (E[IT]) (Fig. $1 E ; t$ test, $\left.t_{(9)}=11.7, p<10^{-6}\right)$. On average, the delay between preparation and initiation was $78 \mathrm{~ms}$, corresponding to a $60 \%$ inflation of the RT above what would be expected if behavior were initiated immediately after becoming appropriately prepared.

\section{Alternative estimate of $T_{P}$}

One potential weakness of the above analysis is that participants could have achieved an advantage by guessing the location of the target and preparing an associated movement but only initiating this movement at the fourth tone if it turned out to be correct (and delaying initiation otherwise). Consequently, success rates might have been inflated at low RTs. Close examination of the distribution of RTs in the Forced RT condition (Fig. $2 F$ ) shows that participants did indeed generate a disproportionate number of movements with long RTs (relative to the uniform distribution of target presentation times imposed), implying that they delayed initiation beyond the fourth tone in a significant number of trials.

To control for this potential confound to our estimate of $T_{\mathrm{P}}$, we developed an alternative method to estimate the timing of movement preparation. We exploited the fact that the distribution of guessed movement directions at very low RTs and in catch trials was not uniform but was biased toward particular targets (Fig. $3 A, B$ ). The specific pattern of movement directions we observed in these trials, dominated by the far-right and near-left target directions, is likely attributable to biomechanical differences in the effort required to move in these different directions (Goble et al., 2007). At RTs between 100 and $150 \mathrm{~ms}$, the distribution of reach directions transitioned from this non-uniform distribution to a uniform one that matched the uniform distribution of actual target locations (Fig. $3 B$ ). The transition between these distributions reflects the time when participants became able to correct their initial guesses in the light of knowledge of the true target location. Critically, this view of the data is not susceptible to the confounding effect of selectively delaying incorrectly guessed movements, which would have led to an inflated success rate at low RTs but would not have affected the distribution of observed reach directions.
The range of RTs over which this transition occurred is broadly consistent with the range of RTs over which success rates improved from chance to accurate (Figs. $1 C, 2 A, C$ ). To estimate the timing of this transition precisely, we adopted a similar logic as before, assuming that movements became appropriately prepared at a random time, $T_{\mathrm{P}}$, and that participants generated a guess (following a specific, non-uniform distribution; Fig. $3 A$ ) if they initiated a movement before this time $\left(\mathrm{RT}<T_{\mathrm{P}}\right)$ and generated an appropriately planned movement otherwise (following a uniform distribution). Pooling data across all 10 participants in Experiment 1, we used maximum likelihood estimation in a sliding $50 \mathrm{~ms}$ window to infer the relative weight of these two distributions (equal to the probability that participants had successfully reprepared any incorrect guesses) as a function of RT (Fig. 3C; for details, see Materials and Methods). We compared this estimate of the cumulative distribution of $T_{\mathrm{P}}$ with our original approach based on success rate (i.e., as in Fig. $2 A, C$, only now using data pooled across all participants). These two different approaches showed a striking agreement with one another (Fig. $3 C$ ). The estimated mean $T_{\mathrm{P}} \mathrm{s}$ (assuming that $T_{\mathrm{P}}$ follows a Gaussian distribution) differed by only $3 \mathrm{~ms}$ (original, $132 \mathrm{~ms}$; alternative, $129 \mathrm{~ms}$ ). The close agreement between these two estimates strongly supports the validity of our original analysis. It also allows us to rule out the possibility that participants gained a significant advantage in the Forced RT task by selectively delaying the timing of movement initiation for incorrect guesses.

\section{Independent timing of movement initiation and movement preparation}

The above analysis demonstrates a significant discrepancy between the timing of movement preparation and the timing of movement initiation. This raises the question of what exactly is the relationship between these two events. One possibility is that participants simply insert a fixed delay between the time at which the movement becomes prepared and the time of initiation (Luce, 1986). Alternatively, there may be no causal relationship between these two events and the timing of movement initiation might be independent of the timing of movement preparation. Such a separation between "what" and "when" is thought to occur for self-generated volitional movements (Brass and Haggard, 2008; Hoffstaedter et al., 2013). These two alternatives, yoked versus independent preparation and initiation, can be dissociated by examining the pattern of spontaneous errors committed in the Free RT condition. If movement initiation is triggered by the end of movement preparation, we should expect errors to be rare and, 

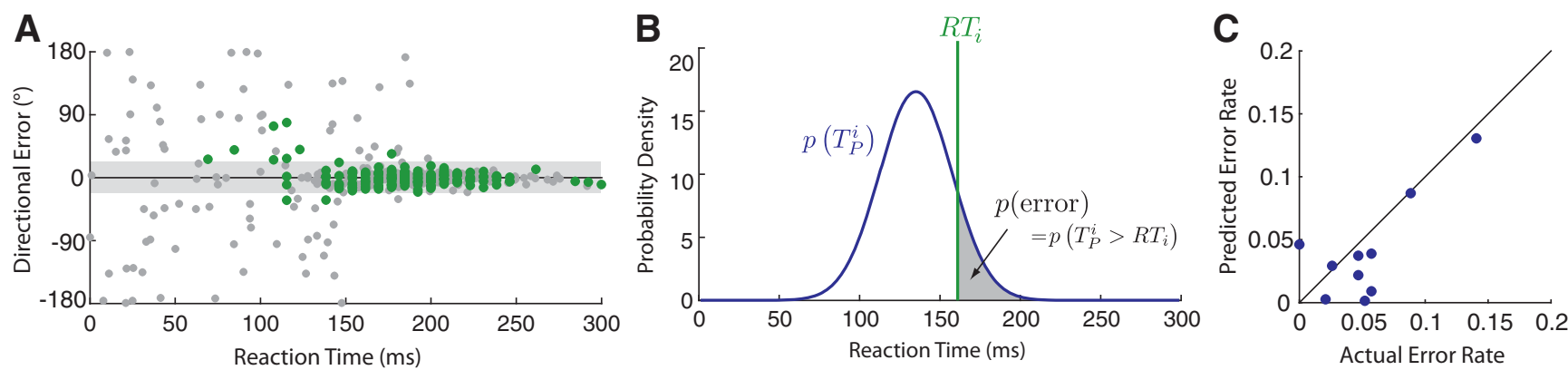

Figure 4. Participants committed spontaneous errors at low RT. $A$, Initial directional error as a function of RT in the Forced RT (gray) and Free RT (green) conditions for a representative participant. $B$, The probability of an error for each trial was predicted based on the estimated distribution of $T_{\mathrm{p}}$ for that participant and the known RT for that trial. $\boldsymbol{C}$, Comparison of actual error rate (total number of errors divided by total number of trials) versus predicted error rate (based on the average probability of an error in each trial, computed as shown in $\boldsymbol{B}$ ).

critically, any errors we do observe ought to be independent of RT. Alternatively, if preparation and initiation are independent, we should expect participants to commit occasional spontaneous errors on trials in which preparation occurred unusually late and/or initiation occurred unusually early. The substantial overlap in the distributions of $T_{\mathrm{P}}$ and $T_{\mathrm{I}}$ in Figure 2, $B$ and $D$, suggests that such errors would be likely to occur at a non-negligible rate.

Closer inspection of behavior in the Free RT condition in Experiment 1 revealed a clear pattern of spontaneous errors that was specific to movements initiated at low RTs (Fig. 4A), supporting the independence hypothesis. To test this hypothesis more quantitatively, we used the distribution of $T_{\mathrm{P}}$ inferred from behavior in the Forced RT condition to predict the rate of spontaneous errors that each participant would commit in the Free RT condition if preparation and initiation truly were independent (Fig. 4B). This model successfully predicted the variability in error rates across participants in the Free RT condition (correlation: $\rho=0.76, p=0.01, r^{2}=0.37$; Fig. $4 C$ ), supporting the hypothesis that preparation and initiation of movement are independent in this task. Under the alternative hypothesis, that preparation and initiation are yoked, spontaneous errors in the Free RT condition ought to be independent of RT and the rate of errors in the Free RT condition should therefore be correlated with the rate of errors seen when participants are forced to move with a long RT in the Forced RT condition (e.g., >300 ms). We observed no such relationship ( $\rho=-0.179, p=0.62)$. In fact, the two participants who exhibited the largest error rates in the Free RT condition had the lowest error rate at high RTs in the Forced RT condition.

This analysis also reinforces our interpretation that the discrepancy in behavior between the Free RT and Forced RT conditions was attributable to delayed movement initiation in the Free RT condition, not slower movement preparation. Our ability to predict error rates in the Free RT condition from behavior in the Forced RT condition implies a consistent relationship between RT and movement preparation (i.e., $T_{\mathrm{P}}$ followed the same distribution) across these two conditions. Therefore, the elevated RTs in the Free RT condition must have been attributable to delayed initiation rather than slower preparation.

\section{Motivation influences initiation but not preparation}

The results of Experiment 1 demonstrate an average $78 \mathrm{~ms}$ discrepancy between the time at which preparation was complete and the time at which movements were actually initiated. One possible explanation for this delay is a simple lack of motivation; the instructions given to participants might not have sufficiently conveyed the importance of moving with as low an RT as possi- ble. Furthermore, although the rate of spontaneous errors was consistent with a model in which movement preparation and movement initiation were independent, the overall rate of errors was very low, and it was therefore difficult to rigorously validate this hypothesis on the strength of these data. We addressed both of these concerns in a second experiment.

In Experiment 2, we motivated participants to initiate movement with as low an RT as possible by imposing a strict deadline on the timing of movement initiation (Pressured RT; Fig. 5A). After an initial block of 96 trials with no constraints on $T_{\mathrm{I}}$ (equivalent to the Free RT condition in Experiment 1), a 900 ms deadline was introduced (for details, see Materials and Methods). Although this time constraint was easily satisfied by all participants, the presence of a time constraint modestly but significantly improved participants' mean RT by an average of $8 \mathrm{~ms}\left(t_{(10)}=\right.$ 2.26, $p<0.05$ ), with no loss of accuracy (error rate, 3.0 vs $2.3 \%$; $\left.t_{(10)}=0.74, p=0.476\right)$. However, the magnitude of this reduction in RT was much less than the $78 \mathrm{~ms}$ difference between preparation and initiation found in Experiment 1. Over five additional blocks, the deadline was progressively reduced to a minimum of $186 \mathrm{~ms}$. Participants responded to the increasing time pressure by progressively reducing their mean RT an additional $60 \mathrm{~ms}$ to a minimum of $146 \mathrm{~ms}$ (Fig. $5 \mathrm{~B}, \mathrm{C}$ ). However, this reduction in RT came at a significant cost to accuracy (Fig. 5D); participants' error rates increased from 3 to $24 \%$. Closer inspection revealed that this increase in error rate was attributable to an increased frequency of trials with very low RT. In addition to a reduction in mean $\mathrm{RT}$, variability of the $\mathrm{RT}$ also increased significantly from Block 2 (900 ms deadline) to Block 6 (186 ms deadline; $t_{(10)}=4.21, p<0.01$; Fig. $5 E$ ).

To test whether movement initiation was independent of movement preparation, participants in Experiment 2 also performed seven blocks of trials under Forced RT conditions (as in Experiment 1). Following the same procedure as for Experiment 1, data from the Forced RT condition allowed us to estimate the relationship between RT and accuracy and, consequently, predict the error rate in each block for each participant under the assumption that preparation and initiation have independent timing. The observed error rates matched these predictions very closely $\left(\rho=0.94, p<10^{-10}, r^{2}=0.86\right.$; Fig. $\left.5 F\right)$, supporting the hypothesis that movement initiation and movement preparation are independent. Furthermore, these results imply that, although the presence of a deadline significantly altered RTs, it had little or no effect on the timing of movement preparation. This selective influence of motivation on initiation but not preparation further supports the notion that these processes are determined by distinct underlying processes. 

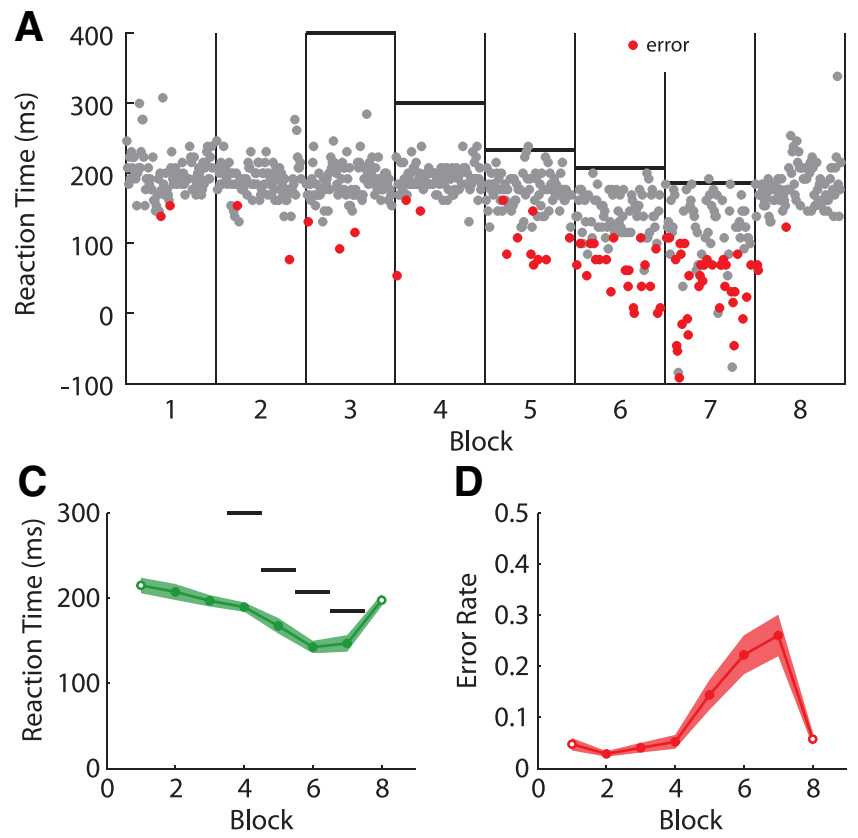

D

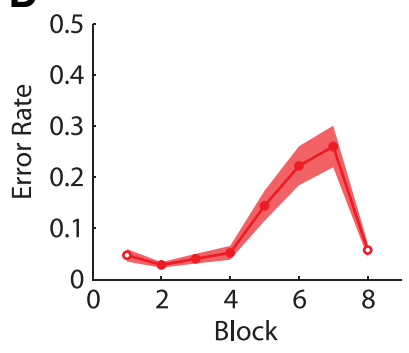

B

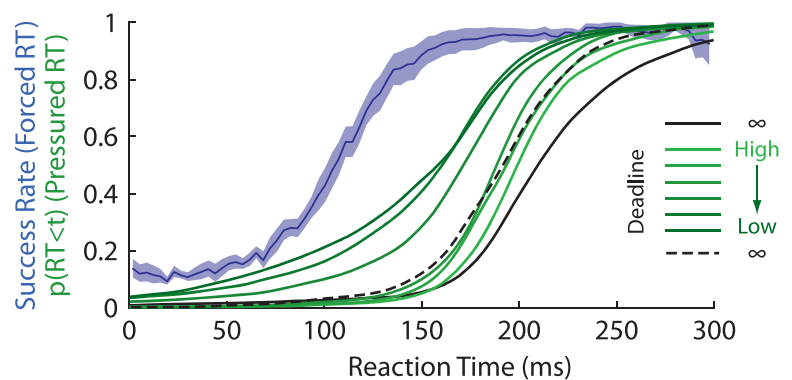

E
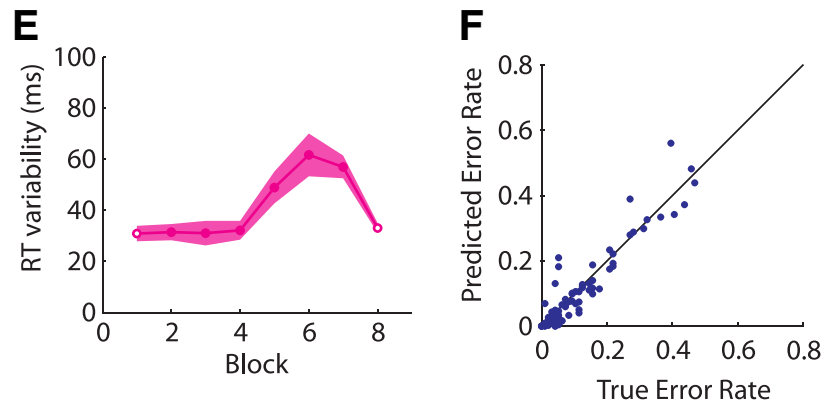

Figure 5. Motivation influenced RT but not $T_{\mathrm{p}} . A, \mathrm{RT}$ as a function of trial number in each block for a representative participant. Gray circles indicate accurately initiated movements. Red circles indicate errors. Vertical gray lines indicate block boundaries. Horizontal black lines indicate initiation deadline. $\boldsymbol{B}$, Blue line, Moving average of the probability of accurate movement initiation as a function of RT, determined from the Forced RT condition. Shaded region indicates \pm SEM across 12 participants. Black lines indicate cumulative RT distribution in first and last (Free RT) blocks (solid, first; dashed, last). Green lines indicate cumulative RT distribution in each block in the Pressured RT condition (lighter, longer deadline; darker, shorter deadline). C, Mean RT across participants. Open circles indicate blocks with no RT deadline. Filled circles indicate blocks with a deadline. Black horizontal lines indicate deadlines (where this fits within axes). Shaded regions indicates \pm SEM across participants. D, Error rate. E, Variability in RT. F, True versus predicted error rate for each block, for each participant.

\section{Discussion}

We have shown that human participants are capable of generating an accurate movement within $\sim 130 \mathrm{~ms}$ of presentation of a visual target. This time course is consistent with the latency at which participants can make corrections to ongoing movements and with the latency at which monkeys can use perceptual information to update the goal of a saccade (Stanford et al., 2010). In contrast, this time course of preparation is significantly faster than would be suggested by participants' voluntary RTs.

Our results demonstrate a clear dissociation between preparation and initiation of movement. These two processes appear to be independent, both in the sense that they are not mechanistically coupled (one can initiate a movement whenever one wants to, regardless of whether it has been appropriately prepared or not) and in the sense that their exact timings are statistically independent of one another. This represents a significant departure from the traditional view of the RT as the total summed duration of component processes that must occur before movement (Donders, 1969; Sternberg, 1969a; Smeets et al., 2015).

It has been noted previously that the RT for movement initiation is substantially longer than the latency to correct a perturbation to an ongoing movement (Hening et al., 1988; Smeets et al., 2015). Previous explanations for this discrepancy suggest that it is attributable to the costly nature of selecting a specific target to be the goal of a movement (Smeets et al., 2015), a cost not incurred when correcting a mid-movement perturbation. However, this theory cannot account for the differences in behavior we observed between the Free RT and the Forced RT conditions because, in both tasks, a single target appeared on a previously blank screen.

Our data instead suggest that the delay between movement preparation and movement initiation is a consequence of partic- ipants' inability to initiate movement at a precise time. Because initiation is independent of preparation, movements are occasionally liable to be initiated before they have been appropriately prepared, resulting in an error (Fig. 4A). Movement initiation appears to be delayed relative to the mean time needed for preparation to avoid the risk of inadvertently initiating a movement before it has been fully prepared.

The separability of preparation and initiation represents an important form of "freedom from immediacy" (Brass and Haggard, 2008) whereby our actions need not necessarily be dictated by our immediate surroundings. Delaying movement initiation beyond the minimum time required for preparation buys additional time in which to alter one's actions based on more declaratively held knowledge (Haith et al., 2015b) or late changes of mind (Resulaj et al., 2009). The ability to initiate movements before they have been appropriately prepared allows evasive action to be taken, however haphazard, in the light of an imminent threat. Many behavioral paradigms designed to test movement preparation and initiation, either by enforcing a delay (Riehle et al., 1994; Churchland et al., 2006) or by controlling RTs with a timed-response paradigm (Schouten and Bekker, 1967; Ghez et al., 1997; Haith et al., 2015a), unwittingly depend on this independence. The most surprising aspect of our findings is the fact that this independence is mandatory; participants were incapable of coupling the timing of movement initiation to the time at which movement preparation became complete, despite the clear incentive to do so.

\section{Implications for volitional versus reactive control}

Theories of behavior often draw a distinction between "reactive" and "volitional" modes of control (Maimon and Assad, 2006; Hallett, 2007; Haggard, 2008; Hoffstaedter et al., 2013; Murakami 
et al., 2014). Reactive movements are those that are triggered directly by an external stimulus, typified by simple reflexes, such as the knee jerk. In contrast, volitional movements are triggered by an internal decision process, such as deciding to pick up a glass to drink. These two modes of control are believed to be supported by distinct neuroanatomy (Haggard, 2008). However, distinguishing reactive and volitional modes of control is difficult because the movements themselves look identical regardless of how they are generated. Consequently, the mode of control used is presumed based on circumstance; tasks in which an imperative stimulus is presented are assumed to engage a reactive mode of control (Jahanshahi et al., 1995; Maimon and Assad, 2006; Perfiliev et al., 2010). In contrast, tasks that only very loosely constrain when an action must be taken are presumed to engage volitional control (Libet, 1993; Maimon and Assad, 2006; Murakami et al., 2014). Our findings suggest the possibility of a more empirical distinction between differing modes of control: based on whether a response to a stimulus is immediate, or occurs only after a delay. Knee jerks and online movement corrections clearly fall into the former category, whereas behavior in our Free RT condition appears to fall into the latter. We speculate that this delay might be a signature of volitional control, reflecting the fact that the stimulus does not directly trigger the movement, but instead triggers an internal decision whether to make a movement.

\section{Potential neural basis of preparation and initiation}

The dissociation between preparation and initiation of movements implies that the two processes occur through distinct neural mechanisms. The rapid improvement in performance we observed with increasing RT closely parallels the time course of neural activity in the primary and premotor cortices during preparation of reaching movements (Riehle and Requin, 1989; Cisek and Kalaska, 2005; Churchland et al., 2006; Ames et al., 2014). Specifically, during an instructed delay, cortical activity appears to converge from an initial default state to a state that is specific to the movement that is about to be made. This shift in cortical activity occurs over a period of $\sim 50 \mathrm{~ms}$, entirely consistent with the timescale of the speed-accuracy tradeoff in our data. Therefore, we suggest that preparatory neural activity in the motor cortex directly relates to the preparation component of our model. The fact that movement initiation is independent of this preparatory process explains why preparatory activity in the motor cortex is only weakly predictive of variability in RT (Afshar et al., 2011; Michaels et al., 2015). This independence also explains why allowing advance preparatory activity during a delay period leads to only modest ( $\sim 30 \mathrm{~ms}$ ) improvements in RT (Churchland et al., 2006; Ames et al., 2014) — because the bulk of the RT is attributable to signaling delays and slow initiation rather than preparation.

A candidate region for the origin of the movement initiation signal is the supplementary motor area, which has long been implicated in the decision to initiate volitional movements (Eccles, 1982; Jahanshahi et al., 1995; Desmurget and Sirigu, 2012). Intriguingly, this area has also been implicated in movement initiation in stimulus-driven tasks (Cunnington et al., 2002), consistent with the idea that even these tasks engage a volitional mode of control.

\section{Implications for the use of RT as a dependent behavioral measure}

Differences in RT have been used for decades to characterize the processes underlying a broad range of cognitive phenomena, in- cluding learning (Knopman and Nissen, 1991), information processing (Sternberg, 1969a), decision-making (Gold and Shadlen, 2001), and memory retrieval (Sternberg, 1969b). Our findings raise important concerns about such approaches. Differences in RT might not necessarily correspond to differences in one's ability at a task (i.e., the speed of preparation) but might instead be attributable to inserting or eliminating unnecessary delays in initiation. Conversely, it is possible that true improvements in ability at a task (i.e., earlier preparation) might be masked if initiation times are not concomitantly reduced. These possibilities can only be disambiguated through the use of paradigms that directly manipulate RTs, as in the experiments presented here.

\section{Implications for neurological disorders}

A better understanding of how we respond to external stimuli might also provide insights into neurological conditions associated with slowed responses, such as Parkinson's disease. Slowed responses in Parkinson's disease could reflect a deficit in either movement preparation (Jahanshahi et al., 1992) or movement initiation (Jahanshahi et al., 1992; Carlsen et al., 2013). Our findings underscore the plausibility of this latter interpretation.

Fast errors, similar to those we saw in the Free RT condition (Fig. 4) are known to be robustly elicited under conditions of stimulus-response incompatibility, such as in the Simon task (Simon et al., 1981; van den Wildenberg et al., 2010) in which people are liable to act based on the location of an instructing stimulus rather than based on the content of the instruction. Such spontaneous, fast errors are often thought to reflect a failure to inhibit default, rapid responses (van den Wildenberg et al., 2010) and are often considered a signature of poor inhibitory control (Davidson et al., 2006; van den Wildenberg et al., 2010). Our findings suggest an alternative interpretation: that such fast errors are in fact attributable to variability in the timing of movement initiation and that movements that happen to be initiated earlier are biased toward default responses.

In conclusion, we suggest that, whenever there is variability in RTs across tasks or neurological conditions, it is important to consider the possibility that this variability is attributable to differences in initiation rather than preparation.

\section{References}

Afshar A, Santhanam G, Yu BM, Ryu SI, Sahani M, Shenoy KV (2011) Single-trial neural correlates of arm movement preparation. Neuron 71: 555-564. CrossRef Medline

Ames KC, Ryu SI, Shenoy KV (2014) Neural dynamics of reaching following incorrect or absent motor preparation. Neuron 81:438-451. CrossRef Medline

Brass M, Haggard P (2008) The what, when, whether model of intentional action. Neuroscientist 14:319-325. CrossRef Medline

Carlsen A, Chua R, Inglis JT, Sanderson DJ, Franks IM (2004) Prepared movements are elicited early by startle. J Mot Behav 36:253-264. CrossRef Medline

Carlsen AN, Almeida QJ, Franks IM (2013) Using a startling acoustic stimulus to investigate underlying mechanisms of bradykinesia in Parkinson's disease. Neuropsychologia 51:392-399. CrossRef Medline

Carpenter RH (1999) A neural mechanism that randomises behaviour. J Conscious Stud 6:13.

Churchland MM, Yu BM, Ryu SI, Santhanam G, Shenoy KV (2006) Neural variability in premotor cortex provides a signature of motor preparation. J Neurosci 26:3697-3712. CrossRef Medline

Cisek P (2006) Integrated neural processes for defining potential actions and deciding between them: a computational model. J Neurosci 26: 9761-9770. CrossRef Medline

Cisek P, Kalaska JF (2005) Neural correlates of reaching decisions in dorsal premotor cortex: specification of multiple direction choices and final selection of action. Neuron 45:801-814. CrossRef Medline 
Cunnington R, Windischberger C, Deecke L, Moser E (2002) The preparation and execution of self-initiated and externally-triggered movement: a study of event-related fMRI. Neuroimage 15:373-385. CrossRef Medline

Davidson MC, Amso D, Anderson LC, Diamond A (2006) Development of cognitive control and executive functions from 4 to 13 years: evidence from manipulations of memory, inhibition, and task switching. Neuropsychologia 44:2037-2078. CrossRef Medline

Day BL, Lyon IN (2000) Voluntary modification of automatic arm movements evoked by motion of a visual target. Exp Brain Res 130:159-168. CrossRef Medline

Desmurget M, Sirigu A (2012) Conscious motor intention emerges in the inferior parietal lobule. Curr Opin Neurobiol 22:1004-1011. CrossRef Medline

Donders FC (1969) On the speed of mental processes. Acta Psychol (Amst) 30:412-431. CrossRef

Eccles JC (1982) The initiation of voluntary movements by the supplementary motor area. Arch Für Psychiatr Nervenkrankh 231:423-441.

Erlhagen W, Schöner G (2002) Dynamic field theory of movement preparation. Psychol Rev 109:545-572. CrossRef Medline

Franklin DW, Wolpert DM (2008) Specificity of reflex adaptation for taskrelevant variability. J Neurosci 28:14165-14175. CrossRef Medline

Frith CD, Done DJ (1986) Routes to action in reaction time tasks. Psychol Res 48:169-177. CrossRef Medline

Ghez C, Favilla M, Ghilardi MF, Gordon J, Bermejo R, Pullman S (1997) Discrete and continuous planning of hand movements and isometric force trajectories. Exp Brain Res 115:217-233. CrossRef Medline

Goble JA, Zhang Y, Shimansky Y, Sharma S, Dounskaia NV (2007) Directional biases reveal utilization of arm's biomechanical properties for optimization of motor behavior. J Neurophysiol 98:1240-1252. CrossRef Medline

Gold JI, Shadlen MN (2001) Neural computations that underlie decisions about sensory stimuli. Trends Cogn Sci 5:10-16. CrossRef Medline

Gritsenko V, Yakovenko S, Kalaska JF (2009) Integration of predictive feedforward and sensory feedback signals for online control of visually guided movement. J Neurophysiol 102:914-930. CrossRef Medline

Haggard P (2008) Human volition: towards a neuroscience of will. Nat Rev Neurosci 9:934-946. CrossRef Medline

Haith AM, Huberdeau DM, Krakauer JW (2015a) Hedging your bets: intermediate movements as optimal behavior in the context of an incomplete decision. PLoS Comput Biol 11:e1004171. CrossRef Medline

Haith AM, Huberdeau DM, Krakauer JW (2015b) The influence of movement preparation time on the expression of visuomotor learning and savings. J Neurosci 35:5109-5117. CrossRef Medline

Hallett M (2007) Volitional control of movement: the physiology of free will. Clin Neurophysiol 118:1179-1192. CrossRef Medline

Hening W, Vicario D, Ghez C (1988) Trajectory control in targeted force impulses. IV. Influences of choice, prior experience and urgency. Exp Brain Res 71:103-115. CrossRef Medline

Hoffstaedter F, Grefkes C, Zilles K, Eickhoff SB (2013) The "what" and "when" of self-initiated movements. Cereb Cortex 23:520-530. CrossRef Medline

Jahanshahi M, Brown RG, Marsden CD (1992) Simple and choice reaction time and the use of advance information for motor preparation in Parkinson's disease. Brain J Neurol 115:539-564. CrossRef

Jahanshahi M, Jenkins IH, Brown RG, Marsden CD, Passingham RE, Brooks DJ (1995) Self-initiated versus externally triggered movements. I. An investigation using measurement of regional cerebral blood flow with PET and movement-related potentials in normal and Parkinson's disease subjects. Brain J Neurol 118:913-933. CrossRef

Knopman D, Nissen MJ (1991) Procedural learning is impaired in Huntington's disease: evidence from the serial reaction time task. Neuropsychologia 29:245-254. CrossRef Medline

Libet B (1993) Unconscious cerebral initiative and the role of conscious will in voluntary action. In: Neurophysiology of consciousness, pp 269-306. Contemporary Neuroscientists. Boston: Birkhäuser Boston.

Luce RD (1986) Response times. New York: Oxford UP.

Maimon G, Assad JA (2006) A cognitive signal for the proactive timing of action in macaque LIP. Nat Neurosci 9:948-955. CrossRef Medline

Michaels JA, Dann B, Intveld RW, Scherberger H (2015) Predicting reaction time from the neural state space of the premotor and parietal grasping network. J Neurosci 35:11415-11432. CrossRef Medline

Murakami M, Vicente MI, Costa GM, Mainen ZF (2014) Neural antecedents of self-initiated actions in secondary motor cortex. Nat Neurosci 17:1574-1582. CrossRef Medline

Nashed JY, Crevecoeur F, Scott SH (2014) Rapid online selection between multiple motor plans. J Neurosci 34:1769-1780. CrossRef Medline

Perfiliev S, Isa T, Johnels B, Steg G, Wessberg J (2010) Reflexive limb selection and control of reach direction to moving targets in cats, monkeys, and humans. J Neurophysiol 104:2423-2432. CrossRef Medline

Pruszynski JA, Kurtzer I, Scott SH (2008) Rapid motor responses are appropriately tuned to the metrics of a visuospatial task. J Neurophysiol 100: 224-238. CrossRef Medline

Resulaj A, Kiani R, Wolpert DM, Shadlen MN (2009) Changes of mind in decision-making. Nature 461:263-266. CrossRef Medline

Riehle A, Requin J (1989) Monkey primary motor and premotor cortex: single-cell activity related to prior information about direction and extent of an intended movement. J Neurophysiol 61:534-549. Medline

Riehle A, MacKay WA, Requin J (1994) Are extent and force independent movement parameters? Preparation- and movement-related neuronal activity in the monkey cortex. Exp Brain Res 99:56-74. Medline

Rosenbaum DA (1980) Human movement initiation: Specification of arm, direction, and extent. J Exp Psychol Gen 109:444-474. CrossRef Medline

Schouten JF, Bekker JA (1967) Reaction time and accuracy. Acta Psychol (Amst) 27:143-153. CrossRef Medline

Simon JR, Sly PE, Vilapakkam S (1981) Effect of compatibility of S-R mapping on reactions toward the stimulus source. Acta Psychol (Amst) 47: 63-81. CrossRef Medline

Smeets JB, Oostwoud Wijdenes L, Brenner E (2015) Movement adjustments have short latencies because there is no need to detect anything. Motor Control. Advance online publication. Retrieved February 3, 2016. CrossRef.

Stanford TR, Shankar S, Massoglia DP, Costello MG, Salinas E (2010) Perceptual decision making in less than 30 milliseconds. Nat Neurosci 13: 379-385. CrossRef Medline

Sternberg S (1969a) The discovery of processing stages: extensions of Donders' method. Acta Psychol (Amst) 30:276-315. CrossRef

Sternberg S (1969b) Memory-scanning: mental processes revealed by reaction-time experiments. Am Sci 57:421-457. Medline

Valls-Solé J, Rothwell JC, Goulart F, Cossu G, Muñoz E (1999) Patterned ballistic movements triggered by a startle in healthy humans. J Physiol 516:931-938. CrossRef Medline

van den Wildenberg WP, Wylie SA, Forstmann BU, Burle B, Hasbroucq T, Ridderinkhof KR (2010) To head or to heed? Beyond the surface of selective action inhibition: a review. Front Hum Neurosci 4:222. CrossRef Medline

van Sonderen JF, Denier van der Gon JJ (1991) Reaction-time-dependent differences in the initial movement direction of fast goal-directed arm movements. Hum Mov Sci 10:713-726. CrossRef

Welford A (1980) Choice reaction time: basic concepts. In: Reaction times. New York: Academic.

Wickelgren WA (1977) Speed-accuracy tradeoff and information processing dynamics. Acta Psychol (Amst) 41:67-85. CrossRef

Wong AL, Haith AM, Krakauer JW (2015) Motor planning. Neuroscientist 21:385-398. CrossRef Medline

Yang L, Michaels JA, Pruszynski JA, Scott SH (2011) Rapid motor responses quickly integrate visuospatial task constraints. Exp Brain Res 211: 231-242. CrossRef Medline 